\title{
New cross section determination for secondary cosmic ray electrons and positrons in the light of new data from collider experiments
}

\author{
Luca Orusa, ${ }^{a, b, *}$ Michael Korsmeier, ${ }^{c, d}$ Fiorenza Donato $^{a, b}$ and Mattia Di Mauro ${ }^{b}$ \\ ${ }^{a}$ Dipartimento di Fisica, Università di Torino, \\ Via P. Giuria 1, Torino, Italy \\ ${ }^{b}$ Istituto Nazionale di Fisica Nucleare, Sezione di Torino, \\ Via P. Giuria 1, 10125 Torino, Italy \\ ${ }^{c}$ The Oskar Klein Centre, Department of Physics, Stockholm University, \\ AlbaNova, SE-10691 Stockholm, Sweden \\ d2Institute for Theoretical Particle Physics and Cosmology, RWTH Aachen University, \\ Sommerfeldstr. 16, 52056 Aachen, Germany \\ E-mail: luca.orusa@edu.unito.it
}

The cosmic-ray fluxes of electrons and positrons $\left(e^{ \pm}\right)$are measured with high precision by the space-borne particle spectrometers AMS-02. To infer a precise interpretation of the dominant production process for $e^{ \pm}$in our Galaxy, it is necessary to have a correct description of the secondary component, produced by the interaction of cosmic-ray proton and helium with the interstellar medium. We update the parametrization of the $e^{ \pm}$cross sections in order to obtain a new estimate of the lepton secondary component flux of the cosmic radiation. In the light of new cross section measurements performed at collider experiments of $p+p \rightarrow \pi^{ \pm}+X$, we update the parametrization of the cross sections for these processes and then compute the $e^{ \pm}$ones from $\pi^{ \pm}$decay. We use for the first time in this field the $e^{ \pm}$spectrum obtained from the muon decay computed till the next to leading order. The peculiarity of this work is the experiment based approach, that we adopt in order to obtain a better shape determination and a significant reduction of uncertainty of the current secondary cosmic ray $e^{ \pm}$flux predictions.

$37^{\text {th }}$ International Cosmic Ray Conference (ICRC 2021)

July 12th - 23rd, 2021

Online - Berlin, Germany

\footnotetext{
*Presenter
} 


\section{Introduction}

During the last decades, the space-based spectrometers PAMELA, AMS-02, DAMPE and CALET have performed unprecedented precise measurements of the cosmic ray (CR) fluxes with uncertainties at the percent level in an energy range from $1 \mathrm{GeV}$ to a few $\mathrm{TeV}$, making the physics of charged CRs a precision discipline. They have measured the CR nuclear [1-7] and leptonic (positron and electron, $e^{ \pm}$) [8-12] components, as well as CR antiprotons [13, 14]. The most recent positron flux measurement by AMS-02 extends from 1 to $1000 \mathrm{GeV}$ with an uncertainty $<5 \%$ for almost the whole energy range. The new precise flux data have stimulated numerous analyses on Galactic CR propagation, lepton production in astrophysical environments like pulsars and supernova remnants and particle dark matter annihilation into antimatter (see, e.g., [15-17]). It is generally established that a large part of $e^{ \pm}$in our Galaxy is produced by the interaction of CRs with the interstellar medium (ISM) [18], conventionally called secondary production. Therefore, to infer correct conclusions on any modeling and interpretation of other possible primary sources, an accurate description of this contribution is necessary. The dominant production of secondary flux comes from the proton-proton ( $\mathrm{pp}$ ) channel, namely CR proton on ISM hydrogen, and from the cases with the CR projectile or the ISM target replaced by helium (Hep, pHe, and HeHe). Heavier channels can contribute at the few percent level. The energy-differential cross sections of $e^{ \pm}$, that are mainly generated from the decay of pions $\left(\pi^{ \pm}\right)$and $\operatorname{kaons}\left(K^{ \pm}\right)$produced in these collisions, enter in the secondary source term calculation.

There are two different strategies to parametrize these cross sections. The first possibility is to find an analytic description of the fully-differential and Lorentz invariant cross section of production of $\pi^{ \pm}$and $K^{ \pm}$, performing a fit to cross section data. Then, a Lorentz transformation, an angular integration and the convolution with the $e^{ \pm}$spectrum from $\pi^{ \pm}$and $K^{ \pm}$decays are applied to find the $e^{ \pm}$energy-differential cross section. This strategy was first pursued by [19]. The other option is to use Monte Carlo predictions to extract the required cross section as in [20], strategy that may be easily affected by uncertainties. As outlined in [18], the adoption of the predictions from different models produces a variation in the normalization of the secondary $e^{ \pm}$flux up to a factor of 2 .

Concerning the first approach, the Tan and Ng parameterizations [19] of the production crosssection of $\pi^{ \pm}$and $K^{ \pm}$are still largely used, despite being mostly 40 years old. The reason is that, until recently, the available dataset was limited to data only collected in the sixties and seventies. In the last decades, however, new experimental datasets have become available, for example the NA61/SHINE Collaboration results collected at the CERN Super Proton Synchrotron (SPS) [21], which provided important information for the energies of interest for AMS-02. Given the importance of these nuclear data for new measurements in astroparticle physics, it is necessary a re-evaluation of the leptonic production cross sections in pp, Hep, pHe, and $\mathrm{HeHe}$ collisions in light of this new available information. In this paper we engage ourselves in this task, in order to provide the community with an updated parametrization for the inclusive $e^{ \pm}$production cross section.

\subsection{Spallation cross-section for $\mathrm{p}+\mathrm{p} \rightarrow e^{ \pm}$}

We first shortly review the basic equations and procedure to calculate the cosmic secondary $e^{ \pm}$ source term. The source term $q_{i j}\left(E_{e^{ \pm}}\right)$originating from the interaction of the CR component $i$ on the ISM component $j$ is given by a convolution integral of the energy-differential $e^{ \pm}$production 
cross section $d \sigma_{i j} / d E_{e^{ \pm}}$with the incoming CR flux $\phi_{i}$ and the ISM target density $n_{I S M, j}$ over the CR energy per nucleon $E_{i}$ :

$$
q_{i j}\left(E_{e^{ \pm}}, \vec{x}\right)=4 \pi n_{I S M, j}(\vec{x}) \int \phi_{i}\left(E_{i}\right) \frac{d \sigma_{i j}}{E_{e^{ \pm}}}\left(E_{i}, E_{e^{ \pm}}\right) d E_{i}
$$

Here the factor $4 \pi$ corresponds to the angular integration of an isotropic CR flux. In order to compute the expected flux of secondary $e^{ \pm}$, the energetic of the projectiles, the composition of the targets and their spatial properties are needed. The interstellar medium gas density of a species $j$ is in general a function of the position in the Galaxy $n_{I S M, j}(\vec{x})$. However, an average local value of $n_{I S M, j}$ is used when dealing with $e^{ \pm}$, since the radiative losses limit their propagation scale to few kpc. As for the projectiles, the primary flux of cosmic p (and He) are typically considered in terms of parametrizations or fits to observed fluxes at the Earth [22]. We now focus on the differential cross-section for the production of $e^{ \pm}$, which occurs through a nuclear reaction between two colliding nuclei, generating mainly charged pions and other mesons. $e^{ \pm}$are one of the final products of the decay chain. For the sake of clarity, we present only the formulae for the protonproton collisions. $e^{ \pm}$are mainly produced as the final output of the decay of charged pions and subsequent muons coming from the excitation of a $\Delta$ resonance:

$$
\mathrm{p}+\mathrm{H} \rightarrow X+\Delta
$$

that can decay into different combinations of pions, proton and neutron. Instead, at higher energies the direct production of charged pions and kaons dominates, following the reaction:

$$
\mathrm{p}+\mathrm{H} \rightarrow X+\pi^{ \pm} \quad \mathrm{p}+\mathrm{H} \rightarrow X+K^{ \pm}
$$

and kaons decay into muons (63.56\%), pions (28.01\%) and pions and leptons $(8.42 \%)$ together, which then decay into $e^{ \pm}$as final products of their decay chain. The production processes of $e^{+}$and $e^{-}$are very similar, thought different inclusive cross sections have to be considered. In fact, since both the target and the projectiles involved in the production process are positively charged particles, charge conservation implies that more positrons with respect to electrons are produced in the final states (see [20]). To compute the differential cross section for the $e^{ \pm}$production processes from pions for example, we need the probability of spallation of a proton of kinetic energy $E_{p}$ to produce a pion with energy $E_{\pi^{ \pm}}$and the probability $P\left(E_{\pi^{ \pm}} \rightarrow E_{e^{ \pm}}\right)$of such a pion to eventually decay into a $e^{ \pm}$of energy $E_{e^{ \pm}}$. This second quantity can be computed thanks to quantum electrodynamics.

$$
\frac{d \sigma}{d E_{e^{ \pm}}}\left(E_{p} \rightarrow E_{e^{ \pm}}\right)=\int \frac{d \sigma}{d E_{\pi^{ \pm}}}\left(E_{p} \rightarrow E_{\pi^{ \pm}}\right) \times P\left(E_{\pi^{ \pm}} \rightarrow E_{e^{ \pm}}\right) \times d E_{\pi^{ \pm}}
$$

The cross section for the process involving kaons can be computed in a similar way to the calculation of pion production. However, experiments do not directly measure the energy-differential cross section but the fully-differential cross section, usually expressed in a Lorentz invariant form.

$$
\sigma_{\text {inv }}=E_{\pi^{ \pm}} \frac{d^{3} \sigma}{d p^{3}}\left(\sqrt{s}, x_{R}, p_{T}\right)
$$

where $E_{\pi^{ \pm}}$is the total $\pi^{ \pm}$energy and $p$ its momentum. It is typically a function of the kinematic variables $\sqrt{s}, x_{R}=E_{\pi^{ \pm}}^{*} / E_{\pi^{ \pm}}^{\text {max* }}, p_{T}$, which are the center of mass $(\mathrm{CM})$ energy, the $\pi^{ \pm}$energy 
divided by the maximal $\pi^{ \pm}$energy in the CM frame, and the transverse $\pi^{ \pm}$momentum, respectively. To obtain the energy-differential cross section in Eq. 4, the variables are transferred into the LAB frame, the frame where the target particle is at rest, using a Lorentz transformation. Convenient variables in the LAB frame are the energies of the CR $E_{i}$ and of the pion $E_{\pi^{ \pm}}$and the angle $\theta$ of the produced pion with respect to the incident CR. Finally, the integral over the solid angle $\Omega$ lead to the energy-differential cross sections:

$$
\frac{d \sigma_{i j}}{d E_{\pi^{ \pm}}}\left(E_{i}, E_{\pi^{ \pm}}\right)=p_{\pi^{ \pm}} \int d \Omega \sigma_{\mathrm{inv}}^{(i j)}\left(E_{i}, E_{\pi^{ \pm}}, \theta\right)
$$

\section{2. $e^{ \pm}$spectrum from pion decay}

In this Section we review the principal steps in the computing of the $e^{ \pm}$spectrum from pion decay. For the first time in this field we consider the polarized muon decay in the muon rest frame, computed till the next to leading order term(NLO) in [23], adopting the Fermi theory. Cosmic ray muons origin from pion decay and are then polarized. The $e^{ \pm}$spectrum in the muon rest frame is described by:

$$
F\left(\epsilon_{e^{ \pm}}^{\prime}, \cos \theta^{\prime}\right)=C\left[f\left(\epsilon_{e^{ \pm}}^{\prime}\right) \pm g\left(\epsilon_{e^{ \pm}}^{\prime}\right) \cos \theta^{\prime}\right]
$$

where $g\left(\epsilon_{e^{ \pm}}^{\prime}\right)$ is the term generated by the muon polarization, $\epsilon_{e^{ \pm}}^{\prime}$ is the energy of the $e^{ \pm}$and $\theta^{\prime}$ is the angle between the direction of polarization of the muon and the direction of motion of the $e^{ \pm}$. For the unpolarized muon decay, only $f\left(\epsilon_{e^{ \pm}}^{\prime}\right)$ enters in Eq. 7. As in [24], we perform two Lorentz transformations from the muon rest frame to the pion rest frame and from the pion rest frame to the $\mathrm{LAB} / \mathrm{Galaxy}$ frame (we indicate the $e^{ \pm}$energy in the LAB frame as $\epsilon_{e^{ \pm}}$). In the numerical code GALPROP [25] the $e^{ \pm}$spectrum from pion decay is currently implemented using the Eqs. $36,37,38,39$ reported in [26]. In this work we upgrade their results thanks to the NLO term of the muon decay and without adopting the approximation of $m_{e}=0$. In Fig. 1 we report $\epsilon_{\pi} F\left(\epsilon_{e^{ \pm}}, \epsilon_{\pi}\right)$ obtained for different pion energies in the LAB frame.

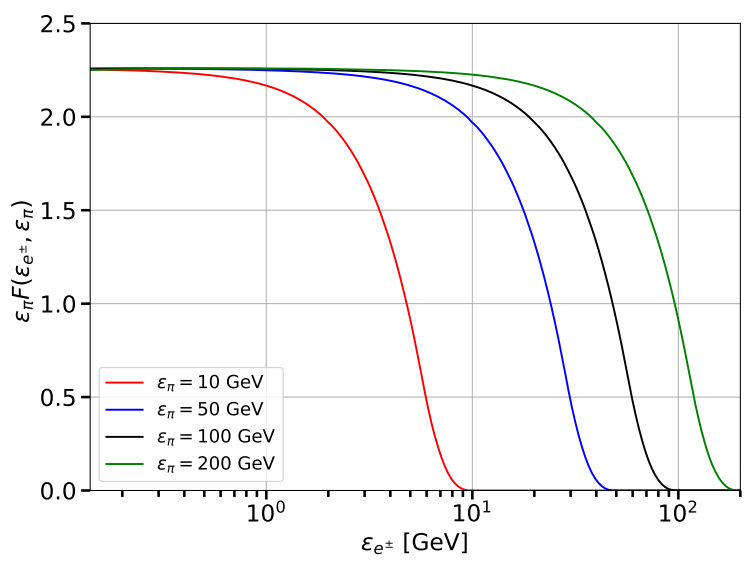

Figure 1: $\epsilon_{\pi} F\left(\epsilon_{e^{ \pm}}, \epsilon_{\pi}\right)$ obtained from the pion and subsequent muon decays for 4 pion different energies. 


\subsection{Fit to the NA61 data}

In this section we report some preliminary results concerning the fit to cross section data. We start our analysis from the measures provided by NA61/SHINE Collaboration [21] and collected at the CERN Super Proton Synchrotron (SPS), that performed recently precise measurements of $\pi^{ \pm}$ and $K^{ \pm}$inclusive cross sections from $\mathrm{p}+\mathrm{p}$ interaction, for different $\sqrt{s}$ values. The experiment kinematic setup is based on the collision between an incident beam and a fixed target, which is exactly the scenario that takes place in the Galaxy. We begin the analysis from the $\pi^{ \pm}$data, testing different possible parametrizations. In [27] a similar procedure, without performing a fit, was realized using the NA49 measurements [28], outlining how the currently available parametrizations for the inclusive pion cross-section do not provide an adequate description of the data. At the moment we are searching for a new satisfying model able to take into account of the $\Delta$ resonance and of the direct production of $\pi^{ \pm}$. In Fig. 2 we report some preliminary results, obtained from the fit to the NA61/SHINE $\pi^{+}$data [21] at $\sqrt{s}=17.3$, grouped in different $p_{T}$ values, using a tester parametrization. For clarity, the data and the theoretical curves at each $p_{T}$ have been multiplied by a factor of $0.6^{n_{p_{T}}}$, where $n_{p_{T}}$ is the integer(starting from 0 ) counting the $p_{T}$, from lower to higher (i.e. for $p_{T}=0.25 \mathrm{GeV} / \mathrm{c}^{2}$ the rescaling is $0.6^{2}$ ).

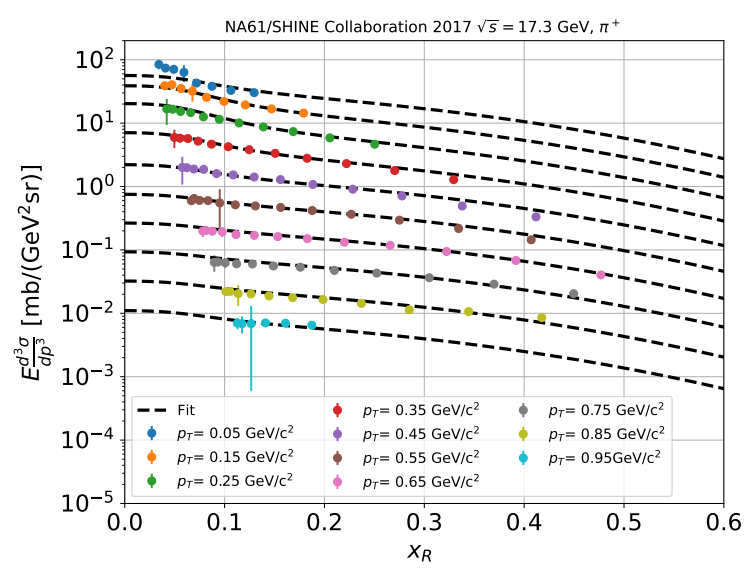

Figure 2: Comparison between NA61 $\pi^{+}$data, grouped in different $p_{T}$ values, with a tester fitting function. For clarity, the data and the theoretical curves at each $p_{T}$ have been multiplied by a factor of $0.6^{n_{p_{T}}}$, where $n_{p_{T}}$ is the integer counting the $p_{T}$, from lower to higher (i.e. for $p_{T}=0.25 \mathrm{GeV} / \mathrm{c}^{2}$ the rescaling is $0.6^{2}$ ).

\section{Conclusions}

We discussed a novel approach in the determination of the $e^{ \pm}$cross section from pp collisions. Our preliminary results show that the method can provide useful information in the computing of the secondary positron production. The next step of our analysis will be the combination between different datasets, considering also measurements from NA49 [28], ALICE [29] and CMS [30]. The procedure will be repeated also for $K^{ \pm}$and for the other possible reactions ( $\mathrm{pHe}, \mathrm{Hep}, \mathrm{HeHe}$ ). Once obtained the parametrizations, we will perform the convolution with the decay spectrum of $e^{ \pm}$from pions and kaons in order to obtain the inclusive $e^{ \pm}$cross section in nuclear reactions that enters in the source term of the secondary flux. 


\section{Acknowledgments}

The work of FD, LO and MK is supported by the DePARTMENTs of ExCELLENCE grant awarded by the Italian Ministry of Education, University and Research (MIUR), the Research grant The Dark Universe: A Synergic Multimessenger Approach, No. 2017X7X85K funded by the MiUR and by the Research grant TAsP (Theoretical Astroparticle Physics) funded by Istituto Nazionale di Fisica Nucleare. M.K is also supported by Istituto Nazionale di Fisica Nucleare (INFN), by the Italian Space Agency through the ASI INFN agreement No. 2018-28-HH.0: "Partecipazione italiana al GAPS - General AntiParticle Spec- trometer', and the grant 'The Anisotropic Dark Universe' No. CSTO161409, funded by Compagnia di Sanpaolo and University of Turin. Furthermore, MK is partially supported by the Swedish National Space Agency under contract 117/19 and the European Research Council under grant 742104. MDM research is supported by Fellini - Fellowship for Innovation at INFN, funded by the European Union's Horizon 2020 research programme under the Marie Skłodowska-Curie Cofund Action, grant agreement no. 754496.

\section{Bibliography}

[1] O. Adriani, G. C. Barbarino, G. A. Bazilevskaya, R. Bellotti, M. Boezio, E. A. Bogomolov, L. Bonechi, M. Bongi, V. Bonvicini, S. Borisov, and et al., Pamela measurements of cosmic-ray proton and helium spectra, Science 332 (Mar, 2011) 69-72.

[2] O. Adriani, G. C. Barbarino, G. A. Bazilevskaya, R. Bellotti, M. Boezio, E. A. Bogomolov, M. Bongi, V. Bonvicini, S. Bottai, A. Bruno, and et al., Measurement of boron and carbon fluxes in cosmic rays with the pamela experiment, The Astrophysical Journal 791 (Jul, 2014) 93.

[3] AMS Collaboration, M. Aguilar et al., Precision Measurement of the Proton Flux in Primary Cosmic Rays from Rigidity $1 \mathrm{GV}$ to $1.8 \mathrm{TV}$ with the Alpha Magnetic Spectrometer on the International Space Station, Phys. Rev. Lett. 114 (2015) 171103.

[4] M. Aguilar, D. Aisa, B. Alpat, A. Alvino, G. Ambrosi, K. Andeen, L. Arruda, N. Attig, P. Azzarello, A. Bachlechner, F. Barao, A. Barrau, L. Barrin, A. Bartoloni, L. Basara, M. Battarbee, R. Battiston, A. Bazo, B. Becker, and P. Zuccon, Precision measurement of the helium flux in primary cosmic rays of rigidities $1.9 \mathrm{gv}$ to $3 \mathrm{tv}$ with the alpha magnetic spectrometer on the international space station, Physical Review Letters 115 (11, 2015) 211101.

[5] AMS Collaboration, M. Aguilar et al., Precision Measurement of the Boron to Carbon Flux Ratio in Cosmic Rays from 1.9 GV to 2.6 TV with the Alpha Magnetic Spectrometer on the International Space Station, Phys. Rev. Lett. 117 (2016), no. 23231102.

[6] Q. An, R. Asfandiyarov, P. Azzarello, P. Bernardini, X. J. Bi, M. S. Cai, J. Chang, D. Y. Chen, H. F. Chen, and et al., Measurement of the cosmic ray proton spectrum from 40 gev to 100 tev with the dampe satellite, Science Advances 5 (Sep, 2019) eaax3793. 
[7] O. Adriani, Y. Akaike, K. Asano, Y. Asaoka, M. Bagliesi, E. Berti, G. Bigongiari, W. Binns, S. Bonechi, M. Bongi, and et al., Direct measurement of the cosmic-ray proton spectrum from 50 gev to 10 tev with the calorimetric electron telescope on the international space station, Physical Review Letters 122 (May, 2019).

[8] O. Adriani, G. C. Barbarino, G. A. Bazilevskaya, R. Bellotti, M. Boezio, E. A. Bogomolov, M. Bongi, V. Bonvicini, S. Borisov, S. Bottai, and et al., Cosmic-ray electron flux measured by the pamela experiment between 1 and 625 gev, Physical Review Letters 106 (May, 2011).

[9] AMS Collaboration, Z. Weng, Towards Understanding the Origin of Cosmic-Ray Positrons, PoS ICRC2019 (2020) 091.

[10] AMS Collaboration, M. Aguilar et al., Towards Understanding the Origin of Cosmic-Ray Electrons, Phys. Rev. Lett. 122 (2019), no. 10101101.

[11] DAMPE Collaboration, Direct detection of a break in the teraelectronvolt cosmic-ray spectrum of electrons and positrons, Nature 552 (Nov, 2017) 63-66.

[12] O. Adriani, Y. Akaike, K. Asano, Y. Asaoka, M. Bagliesi, E. Berti, G. Bigongiari, W. Binns, S. Bonechi, M. Bongi, and et al., Extended measurement of the cosmic-ray electron and positron spectrum from $11 \mathrm{gev}$ to 4.8 tev with the calorimetric electron telescope on the international space station, Physical Review Letters 120 (Jun, 2018).

[13] AMS Collaboration, M. Aguilar et al., Antiproton Flux, Antiproton-to-Proton Flux Ratio, and Properties of Elementary Particle Fluxes in Primary Cosmic Rays Measured with the Alpha Magnetic Spectrometer on the International Space Station, Phys. Rev. Lett. 117 (2016), no. 9091103.

[14] O. Adriani, G. C. Barbarino, G. A. Bazilevskaya, R. Bellotti, M. Boezio, E. A. Bogomolov, L. Bonechi, M. Bongi, V. Bonvicini, S. Borisov, and et al., Pamela results on the cosmic-ray antiproton flux from 60 mev to 180 gev in kinetic energy, Physical Review Letters 105 (Sep, 2010).

[15] Y. Génolini, M. Boudaud, M. Cirelli, L. Derome, J. Lavalle, D. Maurin, P. Salati, and $\mathrm{N}$. Weinrich, New minimal, median, and maximal propagation models for dark matter searches with galactic cosmic rays, arXiv:2103.0410.

[16] M. Di Mauro and M. W. Winkler, Multimessenger constraints on the dark matter interpretation of the fermi -lat galactic center excess, Physical Review D 103 (Jun, 2021).

[17] S. Manconi, M. D. Mauro, and F. Donato, Dipole anisotropy in cosmic electrons and positrons: inspection on local sources, Journal of Cosmology and Astroparticle Physics 2017 (Jan, 2017) 006-006.

[18] T. Delahaye, R. Lineros, F. Donato, N. Fornengo, J. Lavalle, P. Salati, and R. Taillet, Galactic secondary positron flux at the earth, Astronomy \& Astrophysics 501 (May, 2009) 821-833. 
[19] L. C. Tan and L. K. Ng, Parametrization of hadron inclusive cross-sections in p-p collisions extended to very low energies, J. Phys. G 9 (1983) 1289-1308.

[20] T. Kamae, N. Karlsson, T. Mizuno, T. Abe, and T. Koi, Parameterization of Gamma, e+/and Neutrino Spectra Produced by p-p Interaction in Astronomical Environment, Astrophys. J. 647 (2006) 692-708, [astro-ph/0605581]. [Erratum: Astrophys.J. 662, 779 (2007)].

[21] NA61/SHINE Collaboration, A. Aduszkiewicz et al., Measurements of $\pi^{ \pm}, K^{ \pm}, p$ and $\bar{p}$ spectra in proton-proton interactions at 20,31,40,80 and $158 \mathrm{GeV} / \mathrm{c}$ with the NA61/SHINE spectrometer at the CERN SPS, Eur. Phys. J. C 77 (2017), no. 10 671, [arXiv: 1705 . 0246].

[22] A. Cuoco, J. Heisig, L. Klamt, M. Korsmeier, and M. Krämer, Scrutinizing the evidence for dark matter in cosmic-ray antiprotons, Physical Review D 99 (May, 2019).

[23] A. Arbuzov, First-order radiative corrections to polarized muon decay spectrum, Physics Letters B $\mathbf{5 2 4}$ (Jan, 2002) 99-106.

[24] J. H. Scanlon and S. N. Milford, Energy Spectra of Electrons from $\pi-\mu-e$ Decays in Interstellar Space., ApJ 141 (Feb., 1965) 718.

[25] A. W. Strong, I. V. Moskalenko, T. A. Porter, G. Jóhannesson, E. Orlando, and S. W. Digel, The galprop cosmic-ray propagation code, 2009.

[26] S. R. Kelner, F. A. Aharonian, and V. V. Bugayov, Energy spectra of gamma-rays, electrons and neutrinos produced at proton-proton interactions in the very high energy regime, Phys. Rev. D 74 (2006) 034018, [astro-ph/0606058]. [Erratum: Phys.Rev.D 79, 039901 (2009)].

[27] J. W. Norbury and L. W. Townsend, Parametrizations of inclusive cross sections for pion production in proton-proton collisions. ii. comparison to new data, Physical Review D 75 (Feb, 2007).

[28] Inclusive production of charged pions in $p+p$ collisions at $158 \mathrm{gev} / \mathrm{c}$ beam momentum, The European Physical Journal C 45 (Dec, 2005) 343-381.

[29] ALICE Collaboration, J. Adam et al., Measurement of pion, kaon and proton production in proton-proton collisions at $\sqrt{s}=7 \mathrm{TeV}$, Eur. Phys. J. C 75 (2015), no. 5 226, [arXiv: 1504.0002].

[30] A. Sirunyan, A. Tumasyan, W. Adam, E. Asilar, T. Bergauer, J. Brandstetter, E. Brondolin, M. Dragicevic, J. Erö, M. Flechl, and et al., Measurement of charged pion, kaon, and proton production in proton-proton collisions at $s=13$ tev, Physical Review D 96 (Dec, 2017). 